\title{
Ultrasound-guided serratus anterior plane block for analgesia after thoracic surgery
}

\author{
Michael Semyonovi,* \\ Ekaterina Fedorinal,* \\ Julia Grinshpun² \\ Michael Dubilet ${ }^{1}$ \\ Yael Refaely ${ }^{3}$ \\ Leonid Ruderman ${ }^{3}$ \\ Leonid Koyfman' \\ Michael Friger ${ }^{2}$ \\ Alexander Zlotnik' \\ Moti Klein' \\ Evgeni Brotfain' \\ 'Department of Anesthesiology and \\ Critical Care, General Intensive \\ Care Unit, Soroka Medical Center, \\ Ben-Gurion University of the Negev, \\ Beer Sheva, Israel; ${ }^{2}$ Department \\ of Public Health, Faculty of Health \\ Sciences, Ben-Gurion University \\ of the Negev, Beer Sheva, Israel; \\ ${ }^{3}$ Department of Cardiothoracic \\ Surgery, Soroka Medical Center, \\ Ben-Gurion University of the Negev, \\ Beer Sheva, Israel \\ *These authors contributed equally \\ to this work
}

This article was published in the following Dove Medical Press journal: Journal of Pain Research

Background: Patients who undergo surgical procedures that impair the integrity of the chest wall frequently experience extremely severe postoperative pain. Opiates and weaker analgesics, such as nonsteroidal anti-inflammatory drugs (NSAIDs), are not sufficiently effective in achieving control of severe pain and might cause respiratory and gastrointestinal complications. In the past decade, there has been an increased interest in the use of regional nerve blocks for post-thoracoscopy and post-thoracotomy analgesia.

Methods: This is a prospective, randomized, double-blind and single-center study. We recruited 104 patients who underwent elective thoracoscopy. Prior to surgery, the participating patients were randomized into one of two study groups: Group 1- the "standard control group" that received standard postoperative pain control with intravenous opioids, NSAIDs and acetaminophen (paracetamol) and Group 2- the "block group" that was treated by ultrasound-guided serratus anterior plane (SAP) block (a single injection of $0.25 \%$ bupivacaine hydrochloride $2 \mathrm{mg} / \mathrm{kg}$ plus dexamethasone $8 \mathrm{mg}$ ) with standard postoperative pain control regimen. We compared the clinical, laboratory, and postoperative pain assessment data of both groups.

Results: Patients in the SAP block Group 2 reported significantly lower levels of pain after thoracic surgery as assessed by their visual analog scale scores, as compared to the patients in the standard pain control Group $1(P<0.001)$. The total dosage of morphine and tramadol required for pain relief during the first hours after surgery was significantly lower in the patients who received SAP block. Also, the incidence of vomiting after surgery was significantly lower among the patients who received SAP block than among the patients who received standard pain control. Conclusion: The results of the present study suggest that SAP block is an effective adjuvant treatment option for post-thoracic surgery analgesia. Compared to the current methods used for post-thoracic surgery pain relief, SAP block has some significant merits, particularly its ease of use and its low potential for side effects.

Keywords: serratus anterior plane block, thoracic surgery, thoracoscopy, post-thoracic surgery pain relief, ultrasound-guided regional anesthesia, post-thoracotomy analgesia

\section{Introduction}

Patients who undergo surgical procedures that impair the integrity of the chest wall frequently experience extremely severe postoperative pain. ${ }^{1}$ The pain following thoracic surgery often leads to loss of effective coughing due to chest wall splinting with consequent inability to clear secretions. This process can engender serious complications such as pneumonia and respiratory failure, and can also lead to the chronic postthoracotomy pain syndrome. ${ }^{1,2}$ Placement and positioning of intercostal chest tubes after thoracic surgery can also cause severe and even excruciating pain. ${ }^{3}$ The two accepted
Correspondence: Evgeni Brotfain Department of Anesthesiology and Critical Care, Soroka Medical Center, Ben-Gurion University of the Negev, Sderot Rager 15I, Beer Sheva 84I050I, Israel

Tel +972546218106

Emailbem1975@gmail.com 
methods for treating the pain that follows thoracic surgery are opioid drug administration and induction of thoracic epidural anesthesia, but these therapeutic approaches are often associated with serious side effects. ${ }^{4,5}$ Opiates, especially in large doses, can cause respiratory depression ${ }^{6}$ frequently requiring reintubation and reventilation, while local anesthetic spinal procedures such as thoracic epidural blockade (TEB) and paravertebral blockade (PVB) are not only technically difficult to perform and do not always provide optimal pain relief, but they can also entail risks such as inadvertent intravascular injection, total spinal anesthesia and pneumothorax. ${ }^{7}$ Weaker analgesics, such as nonsteroidal anti-inflammatory drugs (NSAIDs), are not sufficiently effective in achieving control of severe pain ${ }^{8}$ and are not infrequently complicated by gastrointestinal bleeding. Similarly, intermediately potent opiates such as tramadol are not usually sufficiently effective in controlling post-thoracotomy and post-thoracoscopy pain.

Consequently, in the past decade, there has been an increased interest in the use of regional nerve blocks for post-thoracoscopy and post-thoracotomy analgesia. ${ }^{4,9,10}$ This is especially related to the introduction of ultrasound guidance, which has facilitated the administration of a variety of plane blocks to achieve effective regional anesthesia. In the present clinical trial, we compared ultrasound-guided serratus anterior plane (SAP) block with traditional early postoperative pain management regimens for patients who have undergone thoracic surgery, based on intravenous (IV) administration of opioids, NSAIDs and paracetamol.

\section{Patients and methods}

This prospective, randomized, double-blind and single-center study was carried out at the Soroka Medical Center, a 1,000bed tertiary care, Trauma Level I teaching hospital located in Beer Sheva in southern Israel. The Human Research and Ethics Committee at Soroka Medical Center approved the study (RN-0345 - 16.SOR). Informed consent was obtained from each participating patient before initiation of the study protocol. During the course of the study, we collected and analyzed the clinical and laboratory data of a total of 104 participating patients who underwent elective thoracoscopy (including cases converted to thoracotomy) at the Soroka Medical Center between December 2016 and January 2018.

\section{Inclusion criteria}

Adult patients aged $\geq 18$ who underwent elective videoassisted thoracoscopic surgery between December 2016 and January 2018 at the Soroka Medical Center and who met the criteria of classes I-III of the American Society of
Anesthesiology (ASA) classification were eligible for inclusion in the study.

\section{Exclusion criteria}

Age $<18$, pregnancy and refusal to participate in the study constituted the exclusion criteria. Also, patients who were unconscious or mentally incompetent prior to surgery, or who were in ASA classes IV or V, or had coagulopathy or hemodynamic instability (systolic blood pressure $<90 \mathrm{mmHg}$, mean arterial pressure $<60 \mathrm{mmHg}$ ), or a history of opioid abuse or of allergic reactions to opiates or to local anesthetic agents were excluded from the study.

\section{SAP block protocol}

Prior to surgery, the participating patients were randomized into one of two study groups (Scheme 1). The two groups were:

- Group 1 (the "standard control group"): These patients received standard postoperative pain control with IV opioids, NSAIDs and acetaminophen (paracetamol).

- Group 2 (the "block group"): Immediately after induction of anesthesia and positioning for surgery, these patients received ultrasound-guided SAP block (a single injection of $0.25 \%$ bupivacaine hydrochloride $2 \mathrm{mg} / \mathrm{kg}$ plus dexamethasone $8 \mathrm{mg}) .{ }^{11-13}$ These patients also received the standard postoperative pain control regimen. We used the Linear 6-13 MHz probe for ultrasound-guided block.

The SAP block was performed while the patient was in a lateral (dorsal decubitus) position with the ipsilateral arm abducted to $90^{\circ} .{ }^{8}$ The injection was administered in the midaxillary line either in the plane between the serratus anterior muscle and the intercostal muscles between the fourth and fifth ribs, or more superiorly in the plane between the serratus muscle and the teres major and latissimus dorsi muscles.

\section{Postoperative data collection}

Following transfer to the post-anesthesia care unit (PACU) and after the patients were awake, extubated and fully conscious, continuous monitoring of pain intensity was begun. During the postoperative period, each patient's pain level was assessed by the visual analog scale (VAS) score for pain: range $0-10(0=$ no pain, $10=$ worst pain ever $)$. The patients were interviewed every 15 minutes following admission to the PACU and thereafter at 1, 4, 8, 12, 24, 48 and 72 hours postoperatively, and also at discharge. Preoperative pain intensity was also recorded so as to provide a more accurate 


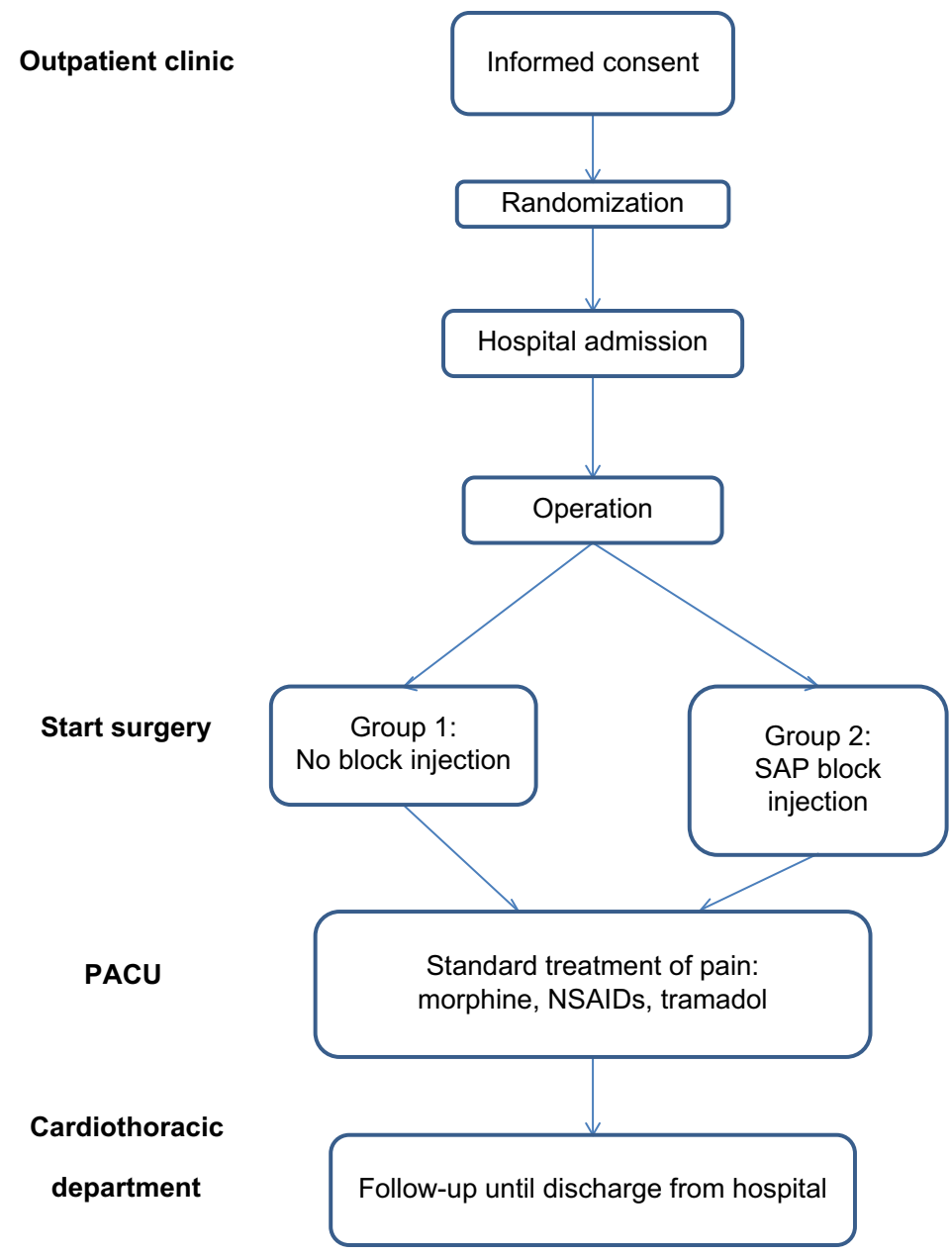

Scheme I Study protocol design.

Abbreviations: NSAID, nonsteroidal anti-inflammatory drug; PACU, post-anesthesia care unit; SAP, serratus anterior plane.

basis for postoperative pain assessment. We administered our routine postoperative pain control regimen consisting of a combination of IV paracetamol in a single dose of $1 \mathrm{~g}$ and IV morphine in incremental doses of $5 \mathrm{mg}$, so as to achieve a VAS score of $0-3$. We recorded all postoperative nausea and vomiting (PONV) events that occurred within the first 24 hours after surgery. Occurrences of urinary retention, constipation and itching were also recorded.

\section{Anesthesia and surgery}

Anesthetic management consisted of fentanyl $(2-3 \mu \mathrm{g} / \mathrm{kg}$ on induction and titrated as needed), isoflurane (minimum alveolar concentration 1-2) and rocuronium bromide for muscle relaxation. Cefazolin was administered for perioperative infection control prior to chest wall incision. All the patients underwent either thoracoscopy or eventual thoracotomy for performance of anatomic or nonanatomic lung resection. The standard thoracoscopy approach in our institution is the three-port technique incorporating two $1 \mathrm{~cm}$ incisions and one $4 \mathrm{~cm}$ utility incision. If thoracoscopy was not feasible because of difficulty in removing a tumor or because of excessive bleeding, thoracotomy was performed. Our standard thoracotomy procedure incorporates a posterolateral approach that entails division of the latissimus dorsi muscle, but spares the serratus anterior muscle.

\section{Variables and measurements}

We collected the following data from the patients' electronic hospital records: demographic information, underlying comorbidities, diagnoses on admission to the cardiothoracic department, duration of the thoracic surgery procedure, duration of the patients' stay in the PACU and duration of their postoperative hospital stay. We also recorded intraoperative findings: the amount of drainage output and the duration of drainage, patients' VAS and PONV scores, occurrence of SAP block complications and therapeutic management 
of the patients during their hospital stay. In addition, the following clinical measurements were recorded: heart rate; arterial blood pressure, respiratory parameters and hourly urine output.

\section{Statistical analysis}

In order to meet the main objective of the study, we calculated samples based on comparison of means using WINPEPI 11.65 software. Sample size was calculated for a significance level of 0.05 and to a power of $90 \%$, with an SD of 2.2 for the control group and 3.6 for the intervention group derived from data from previous studies. A difference of 1.5 between mean VAS scores was considered to be clinically significant. The sample size that matches such data is 100 patients. Data analyses were performed using SPSS (version 16.01 or higher) and were summarized using frequency tables, summary statistics, CIs and $P$-values as appropriate. Continuous variables were compared by Student's $t$-tests. For continuous variables with non-normal distribution, comparisons were evaluated for significance using the Wilcoxon rank sum test. For continuous variables, the $95 \%$ CI was calculated using means and standard errors according to Student's $t$-test statistical hypothesis. Variable selection in multivariable modeling was based on clinical and statistical significance.

\section{Results}

A total of 104 patients (38 males and 66 females) who underwent elective thoracic surgery between December 2016 and January 2018 were recruited into the study. Their mean age was 59 (range 18-70) years. The recruited patients were randomly distributed into one of two study groups (see "Patients and methods" section):

- Group 1: Patients who received standard postoperative pain control (57 patients).

- Group 2: Patients who received ultrasound-guided SAP block for postoperative pain control as well as standard pain control measures as needed (47 patients).

The demographic data and ASA classes of the study patients are summarized in Table 1 . There were relatively more male patients in the SAP block group than in the group that received the standard protocol (Table 1). The two groups were otherwise demographically similar. There was also no difference in the length of hospital stay between the two groups.

Table 2 demonstrates the types of thoracic surgery that were performed and the mean duration of the procedures. All the patients initially underwent thoracoscopy; of these,
Table I Demographic data

\begin{tabular}{|l|l|l|l|}
\hline & $\begin{array}{l}\text { Standard (control) } \\
\text { group }(\mathbf{n = 5 7 )}\end{array}$ & $\begin{array}{l}\text { SAP block } \\
\text { group }(\mathbf{n = 4 7})\end{array}$ & -value $^{\mathbf{a}}$ \\
\hline $\begin{array}{l}\text { Age (years), } \\
\text { mean } \pm \text { SD }\end{array}$ & $56.1 \pm 17.83$ & $62 \pm 14.857$ & 0.57 \\
\hline $\begin{array}{l}\text { Gender } \\
\text { (male), } \mathrm{n}(\%)\end{array}$ & $17(29.8 \%)$ & $21(44 \%)$ & 0.06 \\
\hline Weight $(\mathrm{kg})$ & 71.8 & 72.3 & 0.2 \\
\hline ASA class (n) & & & \\
\hline I & 10 & 7 & 0.1 \\
\hline II & 37 & 32 & 0.8 \\
\hline III & 8 & 8 & 0.9 \\
\hline Ethnicity, n (\%) & & & \\
\hline Jews/Arabs & $48 / 9(84 \% / 15 \%)$ & $43 / 4(91.5 \% / 8.5 \%)$ & 0.3 \\
\hline
\end{tabular}

Note: *A $P$-value of $<0.05$ is considered to be statistically significant. Abbreviations: ASA, American Society of Anesthesiology; SAP, serratus anterior plane.

Table 2 Duration and types of thoracic surgery

\begin{tabular}{|c|c|c|c|}
\hline & $\begin{array}{l}\text { Standard } \\
\text { group } \\
(n=57)\end{array}$ & $\begin{array}{l}\text { SAP block } \\
\text { group } \\
(n=47)\end{array}$ & $P$-value ${ }^{b}$ \\
\hline $\begin{array}{l}\text { Duration of surgery } \\
\text { (minutes), }{ }^{\mathrm{a}} \text { mean } \pm \mathrm{SD}\end{array}$ & $111.54 \pm 15.1$ & $142.67 \pm 10.2$ & 0.04 \\
\hline \multicolumn{4}{|l|}{ Type of surgery $(n)$} \\
\hline Lobectomy & 15 & 17 & 0.8 \\
\hline Segmental (wedge) & 23 & 22 & 0.9 \\
\hline Biopsy & 6 & 4 & 0.9 \\
\hline Exploration & 4 & 1 & 0.9 \\
\hline Pericardial window & I & 2 & 0.9 \\
\hline Decortication & 4 & I & 0.06 \\
\hline Thymomectomy & 2 & I & 0.6 \\
\hline $\begin{array}{l}\text { Converted to } \\
\text { thoracotomy }\end{array}$ & 4 & 5 & 0.7 \\
\hline
\end{tabular}

Notes: ${ }^{2}$ Duration of the procedure including anesthetic preparation. ${ }^{\mathrm{b}} \mathrm{A}$ P-value of $<0.05$ is considered to be statistically significant.

Abbreviation: SAP, serratus anterior plane.

some were eventually converted to thoracotomy. The duration of thoracic surgery including the time spent on anesthetic preparation was significantly longer in the patients who received SAP block than in the control patients who received standard analgesia.

Table 3 shows the pain severity as measured by the patients' VAS scores during their postoperative stay in the PACU and in the cardiothoracic ward. The patients who received SAP block had significantly lower VAS scores during the first eight postoperative hours than the patients who received the standard pain control protocol $(P<0.01)$. From 
Table 3 Comparison of VAS scores in the PACU and in the cardiothoracic surgical department (mean \pm SD)

\begin{tabular}{|l|l|l|l|}
\hline Time & $\begin{array}{l}\text { Standard } \\
\text { group }(\mathbf{n}=57)\end{array}$ & $\begin{array}{l}\text { SAP block } \\
\text { group }(\mathbf{n}=\mathbf{4 7})\end{array}$ & P-value $^{\mathbf{a}}$ \\
\hline $\begin{array}{l}\text { Admission to PACU } \\
\text { (minutes) }\end{array}$ & $0.84 \pm 2.396$ & 0 & $0.0 \mathrm{I}^{\mathrm{a}}$ \\
\hline $\mathrm{I} 5$ & $6.63 \pm 2.288$ & $3.64 \pm 2 . \mathrm{I}$ & $<0.00 \mathrm{I}$ \\
\hline 30 & $5.54 \pm 2.26$ & $2.94 \pm \mathrm{I} .846$ & $<0.00 \mathrm{I}$ \\
\hline 45 & $3.98 \pm 2.279$ & $2.62 \pm \mathrm{I} .824$ & $0.00 \mathrm{I}$ \\
\hline 60 & $2.81 \pm \mathrm{I} .552$ & $2.1 \mathrm{I} \pm \mathrm{I} .28$ & $0.01 \mathrm{I}$ \\
\hline Admission to CTD & $5.0 \pm 2.553$ & $3.4 \pm 2.143$ & $0.00 \mathrm{I}$ \\
\hline 4 hours & $5.05 \pm 2.43 \mathrm{I}$ & $3.04 \pm 2.126$ & $<0.00 \mathrm{I}$ \\
\hline 8 hours & $4.47 \pm 2.00 \mathrm{I}$ & $3.32 \pm \mathrm{I} .878$ & 0.003 \\
\hline I2 hours & $4.77 \pm 2.3 \mathrm{I} 5$ & $3.83 \pm 2.582$ & 0.53 \\
\hline 24 hours & $3.77 \pm \mathrm{I} .58 \mathrm{I}$ & $3.53 \pm 2.175$ & $0.5 \mathrm{I} 7$ \\
\hline Day 2 & $2.86 \pm \mathrm{I} .638$ & $2.62 \pm \mathrm{I} .929$ & 0.848 \\
\hline Day 3 & $\mathrm{I} .28 \pm \mathrm{I} .60 \mathrm{I}$ & $0.98 \pm \mathrm{I} .452$ & $0.32 \mathrm{I}$ \\
\hline Day 4 & $2.55 \pm 2.452$ & $2.43 \pm \mathrm{I} .5 \mathrm{I} 2$ & $0.9 \mathrm{II}$ \\
\hline Day 7 & $\mathrm{I} .20 \pm \mathrm{I} .304$ & $2.00 \pm 0.0$ & 0.450 \\
\hline
\end{tabular}

Note: ${ }^{\text {a } A} P$-value of 0.05 is considered to be statistically significant.

Abbreviations: CTD, cardiothoracic department; PACU, post-anesthesia care unit; SAP, serratus anterior plane; VAS, visual analog scale.

Table 4 Postoperative morphine consumption in the PACU (mg), mean \pm SD

\begin{tabular}{|l|l|l|l|}
\hline $\begin{array}{l}\text { Time } \\
(\text { minutes) }\end{array}$ & $\begin{array}{l}\text { Standard group } \\
(\mathbf{n = 5 7 )}\end{array}$ & $\begin{array}{l}\text { Block group } \\
(\mathbf{n = 4 7 )}\end{array}$ & $\boldsymbol{P}_{\text {-value }}{ }^{\mathrm{a}}$ \\
\hline 0 & $1.84 \pm 3.358$ & $0.98 \pm 2.005$ & 0.128 \\
\hline 15 & $4.12 \pm 2.693$ & $2.57 \pm 2.500$ & 0.03 \\
\hline 30 & $3.04 \pm 2.618$ & $0.8 \pm 1.84$ & $<0.001$ \\
\hline 45 & $0.88 \pm 1.919$ & $0.6 \pm 1.597$ & 0.424 \\
\hline 60 & $0.26 \pm 1.126$ & $0.32 \pm 1.235$ & 0.810 \\
\hline Total dose & $10.14 \pm 5.26$ & $5.48 \pm 4.29$ & $<0.001$ \\
\hline
\end{tabular}

Note: ${ }^{A} A$ P-value of 0.05 is considered to be statistically significant.

Abbreviation: PACU, post-anesthesia care unit.

the ninth postoperative hour onward, there were no significant differences in the VAS scores between the two study groups (Table 3). No difference in VAS scores of two different plane techniques of block, superficial to the serratus muscle or deep to the serratus muscle, was found in Group 2 patients.

Table 4 shows the doses of morphine required for adequate pain relief during the patients' postoperative course. The patients in the SAP block group required a significantly smaller dose of morphine to attain pain relief during their first 30 minutes in the PACU than the patients in the standard protocol group. Table 4 also shows that the total dosage of morphine administered to the patients who received SAP
Table 5 Postoperative tramadol consumption in the PACU (mg), mean \pm SD

\begin{tabular}{|l|l|l|l|}
\hline Time & $\begin{array}{l}\text { Standard group } \\
(\mathbf{n}=57)\end{array}$ & $\begin{array}{l}\text { Block group } \\
(\mathbf{n}=\mathbf{4 7})\end{array}$ & $\boldsymbol{P}^{- \text {value }^{\mathbf{a}}}$ \\
\hline Admission & $3.5 \mathrm{I} \pm 18.564$ & $2.13 \pm 14.564$ & 0.128 \\
\hline 15 minutes & $15.79 \pm 36.718$ & $10.64 \pm 31.166$ & 0.003 \\
\hline 30 minutes & $8.77 \pm 28.540$ & $6.38 \pm 24.709$ & $<0.001$ \\
\hline 45 minutes & $5.26 \pm 22.528$ & $3.80 \pm 18.231$ & 0.424 \\
\hline 60 minutes & 0 & $2.13 \pm 14.586$ & 0.810 \\
\hline Total & $307.01 \pm 23.1$ & $220.21 \pm 25.2$ & 0.02 \\
\hline
\end{tabular}

Note: ${ }^{A} A$ P-value of $<0.05$ is considered to be statistically significant.

Abbreviation: PACU, post-anesthesia care unit.

block was less than the total dosage administered to the standard protocol patients.

Table 5 presents the dosage of tramadol required for postoperative pain relief. As in the case of morphine, the SAP block patients required much smaller doses of tramadol for pain relief than the patients in the standard protocol group.

The total morphine and tramadol doses required for pain relief were found to be significantly lower among the patients who received SAP block than in the patients who received the standard pain control protocol (total morphine dosages $5.48 \pm 4.29$ and $10.14 \pm 5.26 \mathrm{mg}$, respectively $[P<0.0001]$; total tramadol dosages: $220.21 \pm 25.2$ and $307.01 \pm 23.1 \mathrm{mg}$, respectively $[P<0.02])$.

The incidence of postoperative vomiting was found to be less among the patients who received SAP block (two patients [4.2\%]) than among the patients who received the standard protocol (seven patients [12\%]; $P<0.01$ ).

There were no differences in the hemodynamic parameters between the two study groups.

\section{Discussion}

The main findings of the study were: 1) patients in the SAP block group reported significantly lower levels of pain after thoracic surgery as assessed by their VAS scores as compared to the patients in the standard pain control group; 2) the total dosages of morphine and tramadol required for pain relief during the first hours after surgery were significantly lower in the patients who received SAP block than in the patients who received standard pain control; 3) the incidence of vomiting after surgery was significantly lower among the patients who received SAP block than among the patients who received standard pain control and 4) SAP block was not associated with any complications in this study. Of note, there were no statistically significant differences in the hemodynamic and 
respiratory parameters between the patients who received SAP block and those who received standard pain control.

The etiology of acute post-thoracotomy pain has yet to be fully elucidated, but it is believed to result from multiple factors including local damage caused by the skin incision; inflammation of the chest wall structures, for example, osteochondritis; rib injury due to rib retraction and resection during surgery and acute intercostal neuralgia due to damage to the intercostal nerves. ${ }^{14-19}$ The pain experienced after thoracic surgery is one of the most severe types of postoperative pain, and inadequate pain relief can lead to immobility, ineffective breathing and poor clearing of secretions, resulting in an increased incidence of postoperative atelectasis, pneumonia and pulmonary embolism. ${ }^{20}$ The risk of respiratory complications following thoracotomy has been reported to be between $15 \%$ and $32.5 \%,{ }^{21}$ and these respiratory complications are reported to account for more than half of the 30-day mortality following pneumonectomy. ${ }^{22}$ Effective management of acute pain (by regional anesthetic blockade and systemic non-opioid and opioid medicational analgesia) following thoracotomy may help to prevent these complications and may also reduce the likelihood of developing chronic pain.

The IV route is one of the main approaches employed in multimodal analgesia and is used primarily for the administration of opioids. ${ }^{23-25}$ However, IV opioids administered in incremental doses have significant side effects, particularly respiratory depression, sedation, nausea and vomiting, and pruritus. ${ }^{26}$ The results of our study show that the patients in the SAP block group required significantly lower total dosages of morphine and tramadol during their first postoperative hour in the PACU than the patients who received the standard pain control protocol. Moreover, the amount of vomiting after surgery was significantly lower in patients in the SAP block group compared to those in the control group. Most likely, the decrease in the incidence of vomiting resulted from the lower doses of IV opioids administered to the patients who received SAP block. Also, the addition of dexamethasone should have reduced the frequency of vomiting and nausea in Group 2 patients. These findings suggest that the addition of SAP block to opioid administration following thoracic surgery can lead to a significant decrease in the total opioid dose administered postoperatively with a consequent decrease in the incidence of postsurgical complications.

A variety of regional anesthetic blocks are used following thoracic surgery, including TEB, thoracic PVB, intercostal block and intra- or extrapleural block. Each of these blocks has its own specific advantages and disadvantages. TEB in combination with the administration of opioids has been widely regarded as the gold standard for thoracic analgesia and as the best method for achieving a reduction in post-thoracotomy complications. However, this technique requires highly trained medical staff, not only for the insertion and removal of the epidural catheter, but also for the management of the continuous infusion of the pain medication. The risks associated with TEB include accidental dural puncture, inadvertent high block, local anesthetic toxicity and total spinal anesthesia (inadvertent spinal injection of an epidural dose of local anesthetic leading to depression of the cervical spinal cord and the brainstem due to the effects of the local anesthetic on these tissues). Additional side effects such as hypotension, neuraxial hematoma, vomiting and urinary retention have also been reported following TEB. ${ }^{27}$ Furthermore, an epidural puncture is specifically contraindicated in patients who have local infection or a history of previous spinal surgery, or in patients who have coagulation disorders or who are on concurrent anticoagulant or antiplatelet therapy.

PVB is a technique which involves injection of local anesthetic into the paravertebral space to block nerves after they exit the spinal cord. PVB is a unilateral technique, and hence, respiratory and sympathetic functions are preserved on the contralateral side. This feature may be associated with fewer pulmonary complications and less hypotension and urinary retention. ${ }^{19}$ The major potential complications associated with PVB are total spinal block, pneumothorax and neuronal injury. ${ }^{28}$

Intercostal blocks also result in ipsilateral thoracic anesthesia, but the technique demands performance of multiple injections as there is only limited spread of the local anesthetic after a single injection. ${ }^{29}$ Furthermore, multiple intercostal injections are not only painful and time consuming, but also associated with a significant (up to $5.6 \%$ ) incidence of pneumothorax..$^{30,31}$

Due to the side effects and complications of these techniques, alternative methods for palliation of thoracotomy pain are the subject of much current research.

In 2011, Blanco ${ }^{10}$ described a conceptually new type of regional anesthesia, the PECs and PECs II (modified pectoralis muscle blocks, for pain control after breast surgery). Immediately thereafter, numerous studies appeared which not only dealt with the use of pectoralis block in breast surgery, but also described a number of other thoracic blocks for providing regional anesthesia to specific areas of the surface of the thorax. Prominent among these studies were descriptions of SAP block for pain relief of the anteromedial region of the thorax. ${ }^{32-34}$ 
In the present study, we used ultrasound-guided SAP block in the management of post-thoracic surgery acute pain. This block is easy to perform, has a high success rate, and carries minimal complications when performed by an anesthesiologist skilled in ultrasound-guided blocks. In our series, we performed the block when the patients were already anesthetized, and hence, they did not experience any discomfort or pain. Furthermore, SAP block usually requires only a single injection compared to most other regional blocks that often require multiple injections. The duration of thoracic surgery was significantly longer in the patients who received SAP block than in the control patients (mean procedure duration in the block group $142.67 \pm 10.2$ vs $111.54 \pm 15.1$ minutes in the control group $[P=0.04]$ ); but on the other hand, patients benefited by experiencing significantly less pain during the first eight postoperative hours and by requiring lower opioid dosage during that period. There is also some evidence that appropriate analgesia after thoracic surgery can result in earlier extubation, improved ventilatory mechanics and gas exchange, and reduced rates of postoperative atelectasis, pneumonia and pain. ${ }^{31}$

The present study has several limitations. The standard postoperative pain relief regimen in the PACU differed from that in the cardiothoracic ward: in the PACU, the patients received morphine only as needed, while in the cardiothoracic ward, an obligatory pain relief regimen was administered. Furthermore, two technical aspects of SAP block also limit the efficacy of this technique as follows: 1) the zone of anesthesia induced by SAP block sometimes requires the concomitant use of another anesthetic technique and 2) the superficial nature of SAP block does not provide a solution for reduction of pain due to damage to the visceral pleura caused by intercostal drains. There are also some other controversial issues that remain unresolved: SAP block may interfere with the integrity of the serratus muscle, and the fascial plane may be disturbed at the surgical incision site and consequently alter the drug distribution. ${ }^{14,15}$ Moreover, pain after thoracotomy for lobectomy does not match (does not look like) pain after decortication. Pain after decortication has significant skin and muscle components. We should take into account that an SAP block might be inadequate for the muscle component after decortication. Another important issue is the determination of the effective dose of local anesthetic. It appears that SAP block entails a lower risk of local anesthetic toxicity than most other regional anesthetic techniques because the total dose of local anesthetic injected during ultrasound-guided SAP is smaller than that used in the other techniques, and also, the local anesthetic agent is injected into an area which is relatively less vascularized. In the present study, the postoperative VAS scores during the first 8 hours after the surgical procedure were similar in both study groups, signifying that the analgesic effect of the block persisted for up to 8 hours after the patients regained consciousness post-surgery. We suggest future studies to investigate the feasibility of prolonging the effective period of postoperative analgesia induced by SAP block for up to 24 hours by injecting additional doses of local anesthetic as needed or by administering the local anesthetic by the continuous SAP block technique during the postoperative period.

In conclusion, the results of the present study suggest that SAP block is an effective adjuvant treatment option for post-thoracic surgery analgesia. Compared to the current methods used for post-thoracic surgery pain relief, SAP block has some significant merits, particularly its ease of use and its low potential for side effects.

\section{Ethical statement}

The authors declare that the all patients gave written informed consent, and that this study was conducted in accordance with the Declaration of Helsinki. The study was approved by the Soroka University Medical Center Human Research and Ethics Committee (RN-0345 - 16.SOR).

\section{Disclosure}

The authors report no conflicts of interest in this work.

\section{References}

1. Gerner P. Postthoracotomy pain management problems. Anesthesiol Clin. 2008;26(2):355-367.

2. Kolettas A, Lazaridis G. Postoperative pain management. J Thorac Dis. 2015;7(1):62-72.

3. Corcoran JP, Psallidas I, Wrightson JM, Hallifax RJ, Rahman NM. Pleural procedural complications: prevention and management. JThorac Dis. 2015;7(6):1058-1067.

4. Sentürk M, Özcan PE, Talu GK, et al. The effects of three different analgesia techniques on long-term postthoracotomy pain. Anesth Analg. 2002;94(1):11-15.

5. Adcock JJ. Peripheral opioid receptors and the cough reflex. Respir Med. 1991;85 (Suppl 1):43-46.

6. Freise H, van Aken HK. Risks and benefits of thoracic epidural anaesthesia. Br J Anaesth. 2011;107(6):859-868.

7. Gottschalk A, Cohen SP, Yang S, Ochroch EA. Preventing and treating pain after thoracic surgery. Anesthesiology. 2006;104(3):594-600.

8. Kavanagh BP, Katz J, Sandler AN. Pain control after thoracic surgery. A review of current techniques. Anesthesiology. 1994;81(3):737-759.

9. Luketich JD, Land SR, Sullivan EA, et al. Thoracic epidural versus intercostal nerve catheter plus patient-controlled analgesia: a randomized study. Ann Thorac Surg. 2005;79(6):1845-1850.

10. Blanco R. The 'pecs block': a novel technique for providing analgesia after breast surgery. Anaesthesia. 2011;66(9):847-848.

11. Cummings KC, Napierkowski DE, Parra-Sanchez I, et al. Effect of dexamethasone on the duration of interscalene nerve blocks with ropivacaine or bupivacaine. Br J Anaesth. 2011;107(3):446-453. 
12. Fredrickson Fanzca MJ, Danesh-Clough TK, White R. Adjuvant dexamethasone for bupivacaine sciatic and ankle blocks: results from 2 randomized placebo-controlled trials. Reg Anesth Pain Med. 2013;38(4):300-307.

13. Hwang DY, Park SH, Kim SW. A modified dual-plane technique using the serratus anterior fascia in primary breast augmentation. Plast Reconstr Surg Glob Open. 2017;5(2):e1213.

14. Blanco R, Fajardo M, Parras Maldonado T, Parras MT. Ultrasound description of PecS II (modified PecS I): a novel approach to breast surgery. Rev Esp Anestesiol Reanim. 2012;59(9):470-475.

15. Okmen K, Okmen BM, Uysal S. Serratus anterior plane (SAP) block used for thoracotomy analgesia: a case report. Korean J Pain. 2016;29(3):189-192.

16. Khalil AE, Abdallah NM, Bashandy GM, Kaddah TA. Ultrasoundguided serratus anterior plane block versus thoracic epidural analgesia for thoracotomy pain. J Cardiothorac Vasc Anesth. 2017;31(1):152-158.

17. Ökmen K, Ökmen BM. The efficacy of serratus anterior plane block in analgesia for thoracotomy: a retrospective study. JAnesth. 2017;31(4):579-585.

18. Jeng CL, Torrillo TM, Rosenblatt MA. Complications of peripheral nerve blocks. Br J Anaesth. 2010;105(1):i97-i107.

19. Faccenda KA, Finucane BT. Complications of regional anaesthesia incidence and prevention. Drug Saf. 2001;24(6):413-442.

20. Broseta AM, Errando C, De Andrés J, Díaz-Cambronero O, OrtegaMonzó J. Serratus plane block: the regional analgesia technique for thoracoscopy? Anaesthesia. 2015;70(11):1329-1330.

21. Ng A, Swanevelder J. Pain relief after thoracotomy: is epidural analgesia the optimal technique? Br J Anaest. 2007;98(2):159-162.

22. Richardson J, Sabanathan S, Mearns AJ, Evans CS, Bembridge J, Fairbrass M. Efficacy of pre-emptive analgesia and continuous extrapleural intercostal nerve block on post-thoracotomy pain and pulmonary mechanics. J Cardiovasc Surg (Torino). 1994;35(3):219-228.

23. Wang J, Olak J, Ultmann RE, Ferguson MK. Assessment of pulmonary complications after lung resection. Ann Thorac Surg. 1999;67(5):1444-1447.
24. Powell ES, Pearce AC, Cook D, et al. UK pneumonectomy outcome study (UKPOS): a prospective observational study of pneumonectomy outcome. J Cardiothorac Surg. 2009;4(1):41-47.

25. Turktan M, Unlugenc H, Gulec E, Gezer S, Isik G. Coadministration of intravenous remifentanil and morphine for post-thoracotomy pain: comparison with intravenous morphine alone. J Cardiothorac Vasc Anesth. 2015;29(1):133-138.

26. Zhou Y, Huang JX, Lu XH, Zhang YF, Zhang W. Patient-controlled intravenous analgesia for non-small cell lung cancer patient after thoracotomy. J Cancer Res Ther. 2015;11(1):128-130.

27. Takimoto K, Nishijima K, Ono M. Serratus plane block for persistent pain after partial mastectomy and axillary node dissection. Pain Physician. 2016;19(3):481-486.

28. Michelet P, Guervilly C, Hélaine A, et al. Adding ketamine to morphine for patient-controlled analgesia after thoracic surgery: influence on morphine consumption, respiratory function, and nocturnal desaturation. Br J Anaesth. 2007;99(3):396-403.

29. Gulbahar G, Kocer B, Muratli SN, et al. A comparison of epidural and paravertebral catheterisation techniques in post-thoracotomy pain management. Eur J Cardiothorac Surg. 2010;37(2):467-472.

30. Lönqvist PA, Mckenzie J, Soni AK, Conacher AD. Paravertebral blockade: failure rate and complications. Anesthesia. 1995;50:813-815.

31. Moore DC. Intercostal nerve block: spread of India ink injected to the ribs costal groove. Br J Anaesth. 1981;53(4):325-329.

32. Shanti CM, Carlin AM, Tyburski JG. Incidence of pneumothorax from intercostal nerve block for analgesia in rib fractures. J Trauma Acute Care Surg. 2001;51(3):536-539.

33. de Cosmo G, Aceto P, Gualtieri E, Congedo E. Analgesia in thoracic surgery: review. Minerva Anestesiol. 2009;75(6):393-400.

34. Pehora C, Pearson AM, Kaushal A, Crawford MW, Johnston B. Dexamethasone as an adjuvant to peripheral nerve block (review). Cochrane Database Syst Rev. 2017;11:1-112.
Journal of Pain Research

\section{Publish your work in this journal}

The Journal of Pain Research is an international, peer reviewed, open access, online journal that welcomes laboratory and clinical findings in the fields of pain research and the prevention and management of pain. Original research, reviews, symposium reports, hypothesis formation and commentaries are all considered for publication.

\section{Dovepress}

The manuscript management system is completely online and includes a very quick and fair peer-review system, which is all easy to use. Visit http://www.dovepress.com/testimonials.php to read real quotes from published authors. 